\title{
Stacking Sequence Optimisation of Composite Panels Subjected to Slamming Impact Loads using a Genetic Algorithm
}

\begin{abstract}
Optimisation of stacking sequence for composite panels under slamming impact loads using a genetic algorithm method is studied in this paper. For this purpose, slamming load is assumed to have a uniform distribution with a triangular-pulse type of intensity function. In order to perform optimisation based on a genetic algorithm, a special code is written in MATLAB software environment. The optimiser is coupled with the commercial software ANSYS in order to analyse the composite panel under study and calculate the central deflection. After validation, different cases of stacking sequence optimisation are investigated for a variety of composite panels. The investigations include symmetric as well as asymmetric conditions of stacking sequence. Results obtained from these analyses reveal the fact that the adopted approach based on a genetic algorithm is highly capable of performing such optimisations.

\section{Keywords}

Composite Panel; Stacking Sequence; Slamming Impact Load; Optimisation; Genetic Algorithm (GA); Finite Element Analysis (FEA)
\end{abstract}

\section{INTRODUCTION}

Composite materials owing to their lower densities and also higher strength-to-weight ratios are considered to be more advantageous than metallic materials. Besides, composite structures exhibit good resistance against alternate loads, climate effects and especially degradation effects of corrosion. On the other hand, composites are purely non-magnetic materials. All of these properties and characteristics make it reasonable to imagine wide applications for the composite materials in the marine industries. Of course, some of the characteristics of composite materials put different limitations on the way of their applications in real practice. Among such disadvantages or weaknesses, reference may be made to their lower modulus of elasticity and also lower fire resistance. Due to the limitations on the modulus of elasticity for the composite materials, they can not be applied in the construction of long vessels. In spite of all discussed matters, the range of applications of composite 
materials in the construction of high speed marine vehicles is outstanding. In other words, one category of the main structural materials for building high speed crafts is devoted to the composite materials.

Composite panels in the structure of high speed crafts are typically subjected to many different types of in-plane or out-of-plane loads. Among these loads, slamming impact loads are to be considered with a high level of attention. The high speed crafts operate among unsteady sea waves and due to the interactions existing between the crafts and the sea waves, the structural panels of the crafts have to sustain lateral impact loading induced by slamming phenomenon.

It should be noted that the problem of composite panels subjected to lateral impact loads has attracted the attention of many researchers. Of course, most of the research studies are devoted to the structural response analysis of the composite panels under impact by a spherical impactor.

Von Karman [1] was one of the first researchers who investigated the hydrodynamic impact problem by studying seaplane floats during landing. He introduced the concept of added mass due to the water motion induced by the body. This work was followed later by Wagner [2] who considered piled-up water effects. The body was modelled as a flat plate, whose width is determined by the intersection between the elevation of the free surface and the body position. The resulting force is obtained by integrating the pressure distribution. Wagner's work was truly remarkable so that it is still being used in order to estimate the lateral impact loads. In both of these methods, the hydro elasticity effects induced by fluid-structure interactions were ignored. Figure 1 represents a schematic view on the pressure distribution at the event of slamming.

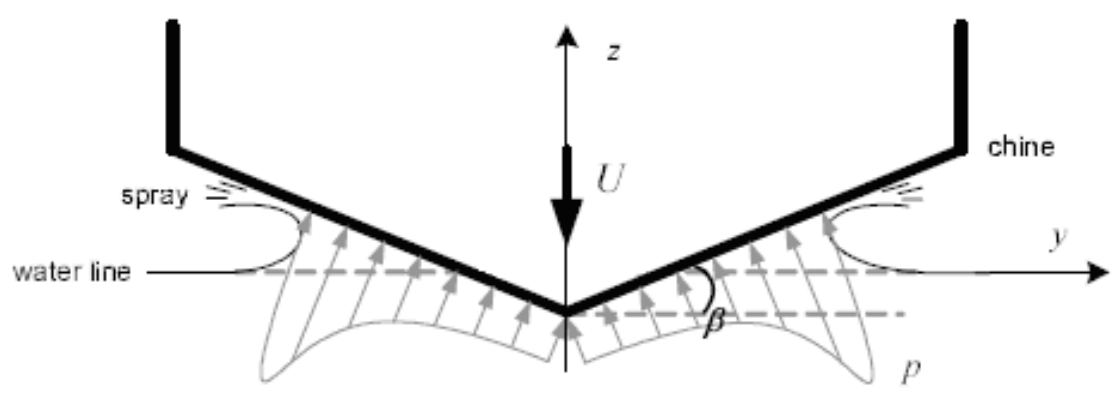

Figure 1 A schematic representation of slamming impact pressure distribution.

It should be emphasised that the slamming is an impulsive time-depending phenomenon in which the pressure distribution changes over the period of time. One interesting model in order to investigate the slamming phenomenon is the one developed by Qin and Batra [3] in which the hydro elasticity effects were also included. As a summary, it can be said that there are numerous models for description of slamming impact load distribution, each of which is based on some assumptions. Depending on the relevant assumptions, any of the models can approximate the slamming load distribution with a corresponding level of accuracy.

Obviously, it is very important to reach the optimum stacking sequences for the plies of these composite panels in order to maximise their resistance against such impact loads. The main goals of optimising a structure against impact are to improve damage resistance, damage tolerance, or ener- 
gy absorption. Damage resistance is a property that enables a structure to resist the onset of damage. Damage tolerance enables a structure to resist the effects of damage. The goal in energyabsorbing designs is to dissipate as much of the kinetic energy as possible.

Genetic algorithms (GAs) are search techniques based on natural selection and population genetics. Selection, crossover and occasionally mutation are applied to a population of candidate solutions sampled from the larger design space, with fitter individuals having a higher probability of reproducing. Genes that predispose certain individuals to have improved fitness are thus sampled more frequently and propagated throughout the population. GAs are robust optimisers that can handle real/discrete variables [4]. Detailed treatments of this ever-growing field may be found in textbooks [5-7].

Callahan and Weeks [8] studied the optimisation of composite laminates using genetic algorithms. Genetic algorithms are nowadays considered as a powerful tool for optimisation of composite panels. Common variables included in such optimisation problems are typically structural dimensions, stacking method, ply materials, ply thicknesses and ply angles. Rahul and Dutta [9] adopted genetic algorithms in order to optimise weight and cost of fibre reinforced composites against impact loads.

Optimisation of stacking sequence for composite panels under slamming impact loads using a genetic algorithm method is studied in this paper. In order to perform optimisation based on a genetic algorithm, a special code is written in MATLAB software environment. The optimiser is coupled with the commercial software ANSYS in an especial procedure that in each optimisation step analyses the composite panel under study and calculates the central deflection. The coupled GAFEA procedure is validated against some available data. Different cases of stacking sequence optimisation are investigated for a variety of composite panels. The investigations include symmetric as well as asymmetric conditions of stacking sequence.

\section{FINITE ELEMENT ANALYSIS}

\subsection{Finite element code and adopted elements for discretisation}

In order to determine the type of finite element analysis of the composite panels studied here, it should be noted that the lateral loads are impulsive and of rapidly varying nature. Therefore, all finite element analyses are to be of transient dynamic type. Besides, large geometric deflections are to be accounted for in the analyses. All of the finite element analyses in this study are carried out using the commercial code ANSYS [10]. SHELL181 elements are selected in order to discretise the composite panels, Fig. 2. These elements have four nodes and six degrees of freedom per node. The formulation of SHELL181 elements is based on first order deformation theory. The reasons behind adopting such elements for finite element analyses in this study include high accuracy of results together with low CPU time.

As a summary, the analyses performed using ANSYS code are of geometrically non-linear transient dynamic type. Full Newton-Raphson approach is included in the solution process for geometrically non-linear analyses. On the other hand, the ANSYS code employs the Newmark method for typical transient dynamic analyses. 


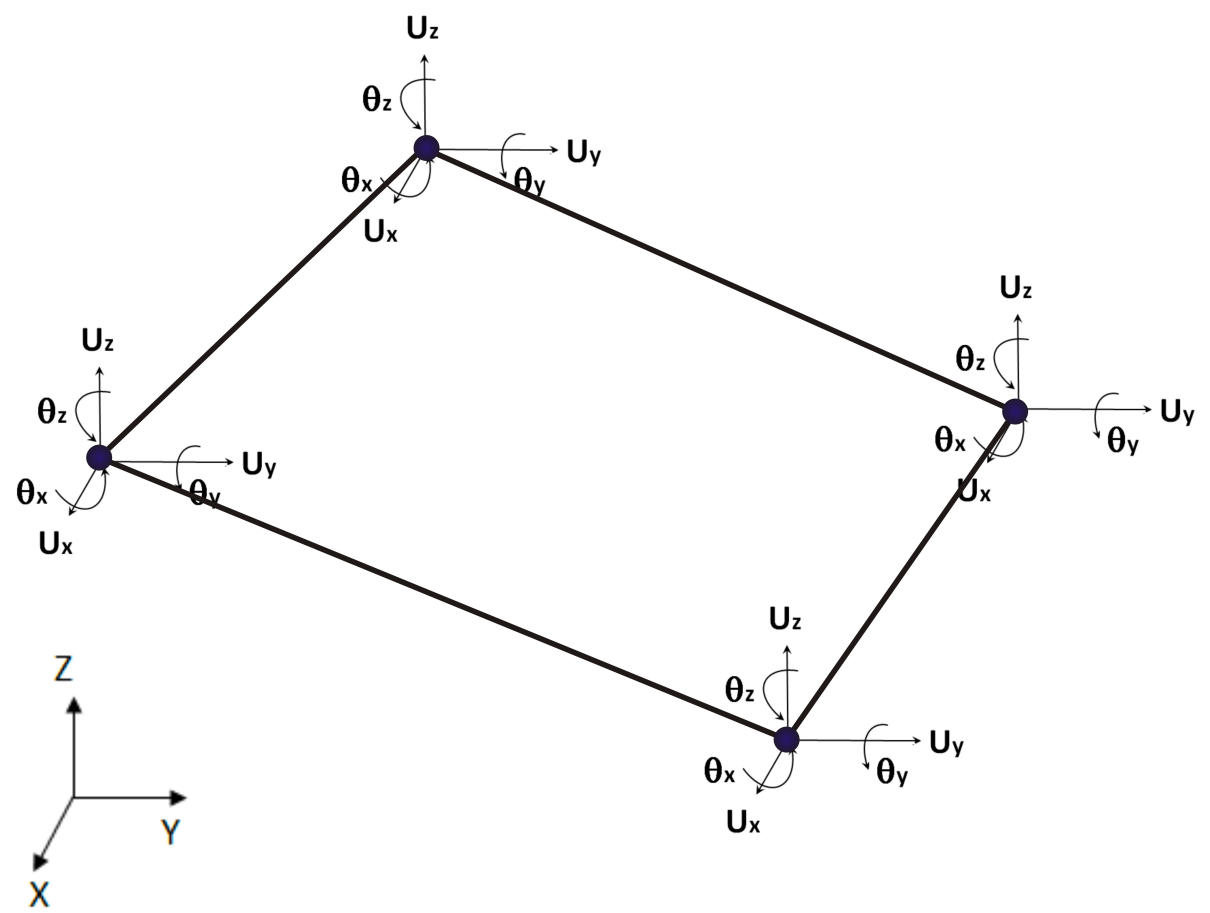

Figure 2 Details of SHELL181 element.

\subsection{Validation of geometrically non-linear transient dynamic analysis procedure}

In order to check the validity of the procedure established in the ANSYS code for performing geometrically non-linear transient dynamic analyses, the model investigated by Kazanc and Mecitoglu [11] is studied here. Kazanc and Mecitoglu studied the nonlinear dynamic response of simply supported laminated composite plates subjected to blast loading. Such a kind of loading is similar in nature to the slamming impact load; both are of rapidly varying type. Kazanc and Mecitoglu derived the governing dynamic equations for the composite plate using the virtual work principle. They applied von Karman large deflection theory in order to consider the geometrical non-linear effects. They also adopted the Friedlander exponential decay equation [12] as follows for describing the distribution of blast load

$$
P(t)=P_{m}\left(1-t / t_{p}\right) e^{-\alpha t / t_{p}}
$$

Geometrical specifications of the panel studied by Kazanc and Mecitoglu, material properties and loading parameters are given in Table 1. A comparison of the result obtained by Kazanc and Mecitoglu with that obtained by present numerical calculations is seen in the Fig. 3. Kazanc and Mecitoglu [11] used the result of Librescu and Nosier [13] in order to verify their result. Figure 3 demonstrates the fact that the result of present numerical analyses lies somewhere between the two sets of results obtained by the other researchers. Small differences existing among the results in Fig. 3 are obviously due to the variations in the adopted formulations or numerical methods. 
Table 1 Geometrical specifications, material and loading properties of the model adopted for validation

\begin{tabular}{|c|c|}
\hline \multicolumn{2}{|l|}{ Geometry of panel } \\
\hline Panel lay-up (Cross ply) & {$[0 / 90 / 0]$} \\
\hline Length of panel (m) & 2.54 \\
\hline Width of panel (m) & 2.54 \\
\hline Thickness of panel (m) & 0.17 \\
\hline \multicolumn{2}{|c|}{ Mechanical properties of material } \\
\hline Longitudinal modulus, $\mathrm{E}_{1}(\mathrm{GPa})$ & 132.4 \\
\hline Transverse modulus, $\mathrm{E}_{2}(\mathrm{GPa})$ & 10.8 \\
\hline Shear Modulus, $\mathrm{G}_{12}(\mathrm{GPa})$ & 5.6 \\
\hline Poisson Ratio, $v_{12}$ & 0.24 \\
\hline Density, $\rho\left(\mathrm{Kg} / \mathrm{m}^{3}\right)$ & 1443 \\
\hline \multicolumn{2}{|l|}{ Load parameters } \\
\hline Maximum load, $P_{m}(\mathrm{KPa})$ & 3447 \\
\hline$t_{p}(\mathrm{Sec})$ & 0.1 \\
\hline$\alpha$ & 2 \\
\hline
\end{tabular}

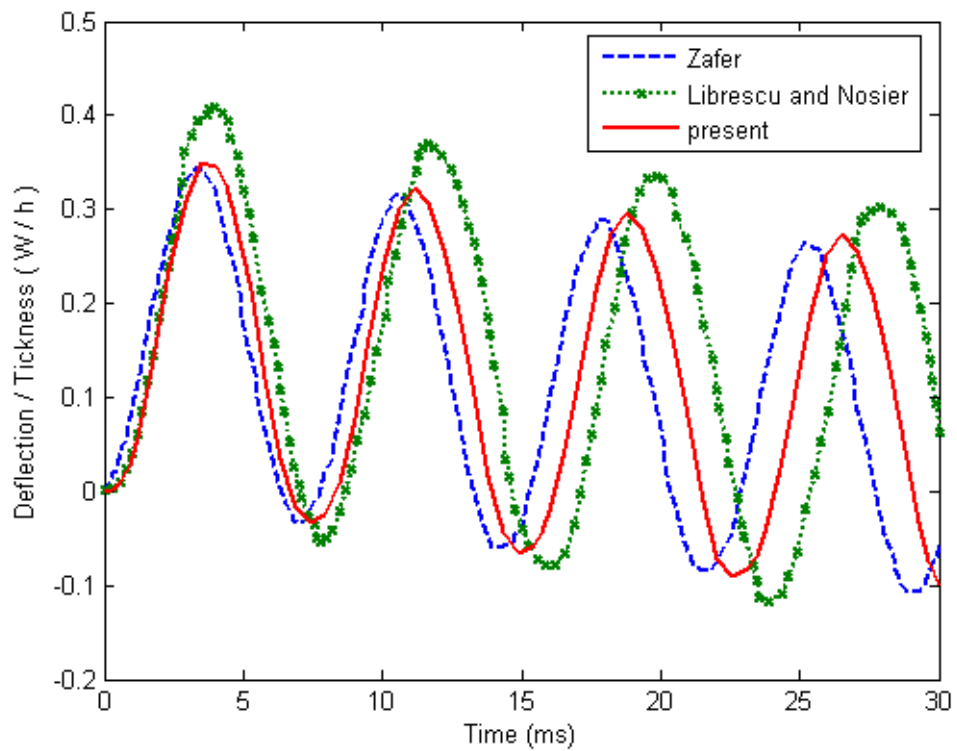

Figure 3 Comparison of present numerical results with those of other researchers. 


\subsection{Dynamic response of a composite panel subjected to slamming impact load}

In this section, the dynamic response of a composite panel under slamming impact load is studied. Table 2 represents the geometry of the panel and relevant mechanical properties which are taken from Aslan et al. [14]. The material of the panel is of E-glass/Epoxy type considering its maritime applications.

Table 2 Geometrical specifications and material properties of the composite panel adopted for optimization studies

\begin{tabular}{cc}
\hline \multicolumn{3}{c}{ Geometry of panel } \\
\hline Panel lay-up & {$[0 / 90 / 0 / 90]$} \\
\hline Length of panel $(\mathrm{m})$ & 1 \\
\hline Width of panel $(\mathrm{m})$ & $0.5,1.0,1.5$ \\
\hline Thickness of panel $(\mathrm{mm})$ & 8 \\
\hline Mechanical properties of material \\
\hline Longitudinal modulus, $\mathrm{E}_{1}(\mathrm{GPa})$ & 44 \\
\hline Transverse modulus, $\mathrm{E}_{2}(\mathrm{GPa})$ & 10.5 \\
\hline Shear Modulus, $\mathrm{G}_{12}(\mathrm{GPa})$ & 5.6 \\
\hline Poisson Ratio, $v_{12}$ & 0.24 \\
\hline Density, $\rho\left(\mathrm{Kg} / \mathrm{m}^{3}\right)$ & 1443 \\
\hline
\end{tabular}

Slamming impact load is distributed all over the surface of the panel and its intensity changes in time according to a triangular pulse diagram as indicated in the Fig. 4. The pulse pressure parameters are taken as $P_{\max }=100 \mathrm{KPa}$ and $T=50 \mathrm{~ms}$. The composite panel is simply supported along its boundaries. Dynamic response of the panel subjected to slamming impact load is shown in the Fig. 5. As can be seen in the Fig. 5, the maximum value of central deflection in the panel is equal to $5.6 \mathrm{~cm}$ that is obtained at a certain time, which is equal to the half of the loading time period. In order to investigate the response of the composite panel after the loading time period, or after slamming load vanishes, the diagram is extended until $t=80 \mathrm{~ms}$. It can be realised that even after unloading of the slamming load, the central deflection of the panel magnifies with progress of time. 


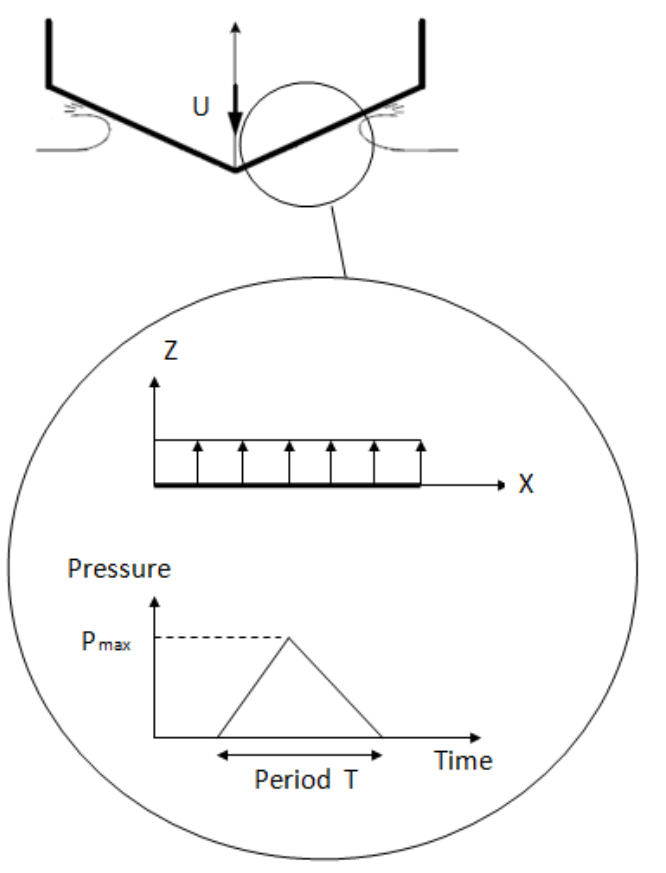

Figure 4 Modelling scheme for slamming impact load.

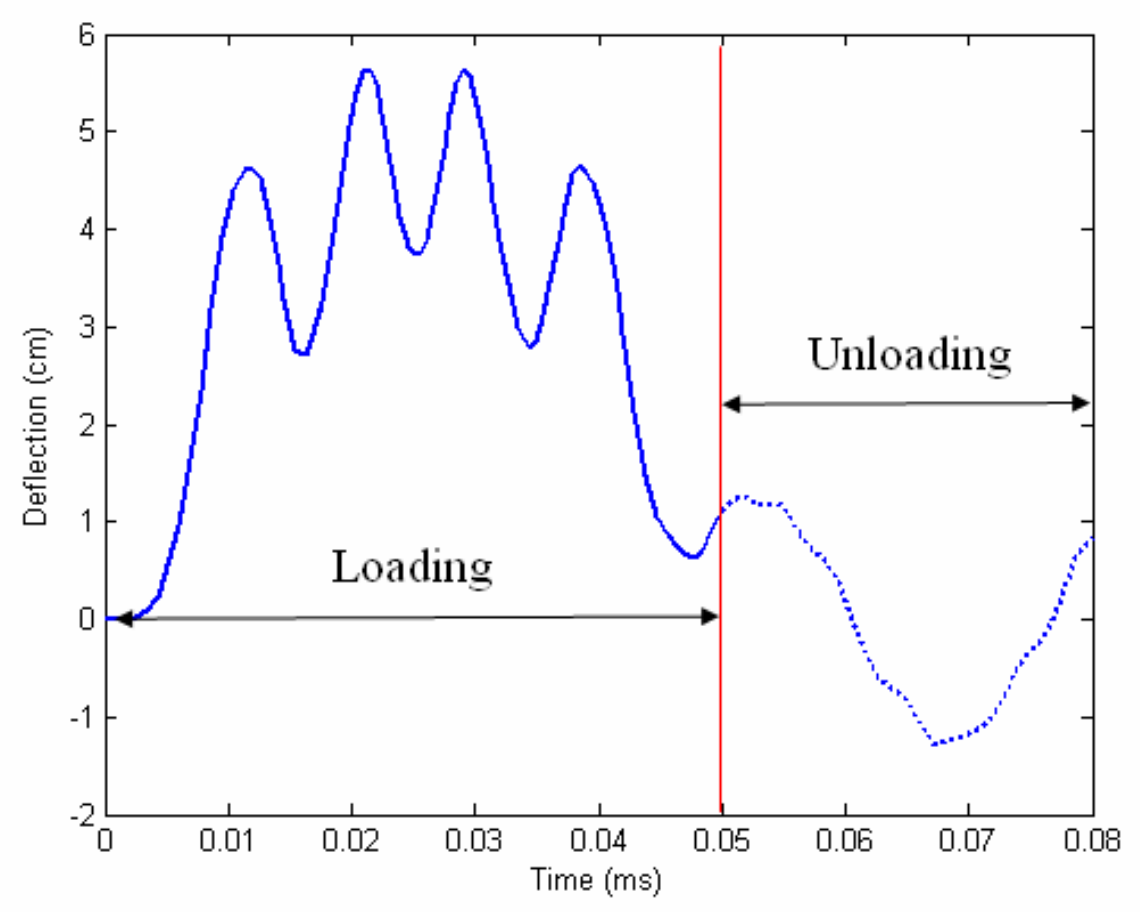

Figure 5 Response of composite panel to slamming impact load. 


\section{OPTIMISATION PROCEDURE USING A GENETIC ALGORITHM}

\subsection{Basic concepts and definitions in genetic algorithms}

In the 1950s and 1960s, several computer scientists independently studied the organizations of evolutionary systems. Their main idea was to check if such evolutionary systems could be applied in the simulation of optimisation problems in real engineering practice. The evolutionary systems were of different types, some had population-based schemes for selection and variation, and some others, like many GAs, had binary strings as abstractions of biological chromosomes. Genetic algorithms are developed by applying the principal of survival of the fittest into a numerical search method [6]. They are used as function optimisers particularly when the variables have discrete values. They achieve this by first selecting an initial population where each individual is constructed by bringing together the total number of variables in a binary or other coded form [6]. These individuals are called artificial chromosomes and they have a finite length string. Every character in the string is an artificial gene, which in the case of binary code has a value of either 1 or 0 . The binary code for each design variable represents the sequence number of this variable in the discrete set.

A genetic algorithm initiates the search for finding the optimum in a discrete space by first selecting the number of individuals randomly and collecting them together to constitute the initial population. It then makes use of four operators to generate a new population. These operators are selection, mating, crossover and mutation. The detailed explanation of these operators is given in Refs. $[6,15,16]$. Among these, the crossover operator is probably the one which plays an important role in the production of the new generation. There are several types of crossover operators such as single point, two-point, multi-point, uniform and variable crossover. It is shown in Ref. [17] that two-point crossover performs much better among the multi-point crossover techniques. The detailed study carried out on the evaluation of crossover techniques has shown that direct design variable exchange produced the best solutions in the test problems considered [18]. Further, operators such as niche and cloning are suggested in Ref. [19].

\subsection{Implementation of a genetic algorithm}

Optimisation of stacking sequence for the plies of the composite panels subjected to slamming impact load is performed in this study using the simple genetic algorithm approach. In order to achieve this, a random initial population of stacking sequence variables in a binary coded form is fed to the algorithm. Finally, as a result of some operations made by the operators of the algorithm, optimum solution for the problem is given as an output. One of the main steps required for each optimisation process, is to determine the fitness of any solution with regard to the optimisation criteria. In other words, it should be investigated how far the obtained solution is located from the optimum solution. In optimisation problems where the aim is to minimise either weight or cost, an analytical equation can be easily derived in order to describe the fitness of solutions. Also, it is possible in some cases that closed-form analytical relationships can not be applied for the fitness evaluation of the solutions. In such cases, numerical methods or algorithms are to be implemented in conjunction with the genetic algorithms. Examples of these are the works reported in $[20,21]$ in

Latin American Journal of Solids and Structures 10(2013) $1043-1060$ 
which coupling of the finite element approach with the genetic algorithms were demonstrated for optimisation of composite structures.

The main aim of optimisation process in the present study is to minimise the central deflection of a composite panel subjected to slamming impact load. It is obvious that simple analytical expressions can not be applied in order to evaluate the fitness of the solutions. Thus, advanced numerical methods are implemented in the optimisation algorithm.

Figure 6 shows the flowchart of the optimisation process based on a genetic algorithm and applying finite element method. The optimisation process consists of two major sections. The first section is devoted to a genetic algorithm. A special genetic algorithm is programmed in the MATLAB environment. Initial population is entered to the genetic algorithm code in the form of random binary strings. The programmed genetic algorithm code has the ability of making changes in the number of chromosomes of the initial population. The governing convergence criterion or stopping condition in the adopted genetic algorithm is assumed as the condition in which the number of generations of the population reaches a certain limit. The value of this limit can be changed depending on the extent of the search space. The so-called roulette wheel method is used in order to perform the function of selection operator in the algorithm. A special capability is considered for the adopted genetic algorithm to be able to use two different crossover operators in an entirely userdefined manner. When the length of chromosomes is relatively short, then the single-point crossover is used as the operator of generation process. This is while; in case of relatively longer chromosomes, three-point crossover is chosen as the operator of generation process.

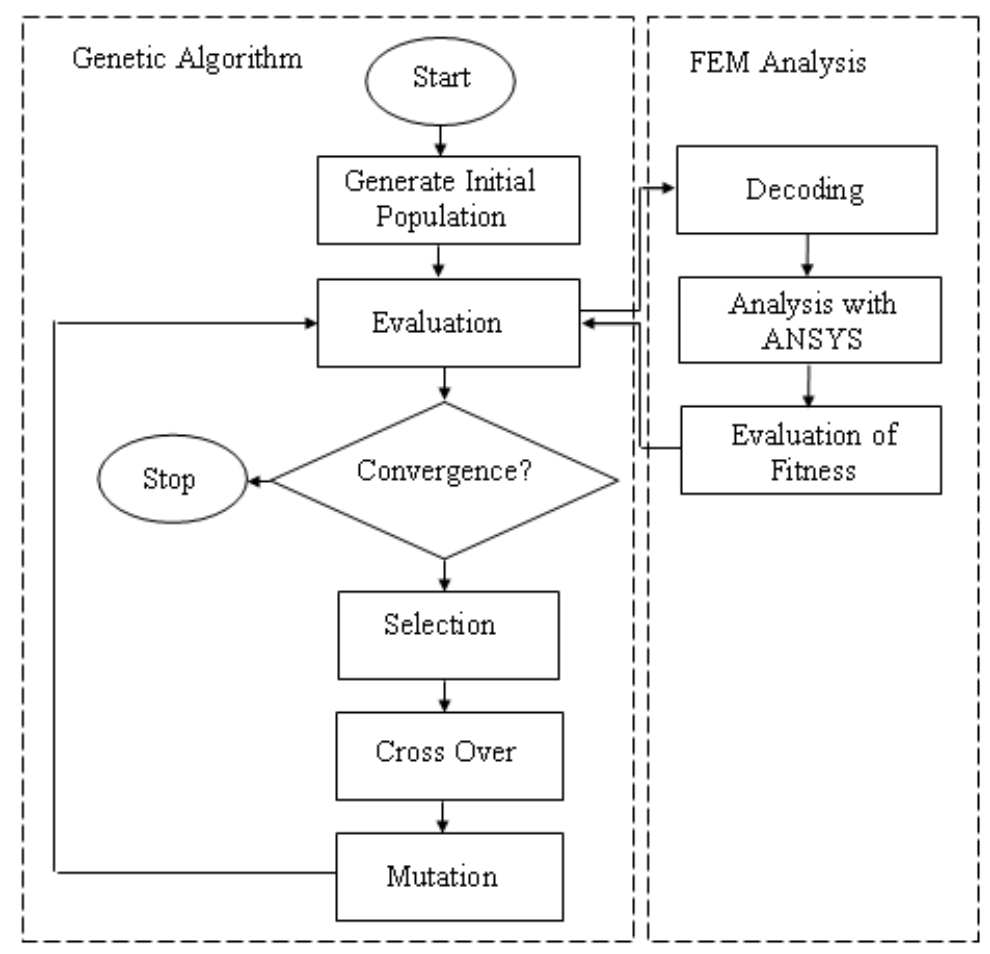

Figure 6 Flowchart of optimisation procedure based on a genetic algorithm and applying finite element method. 
The second section of the optimisation algorithm is devoted to the application of the finite element method in order to control the fitness of chromosomes in the population of each of the generations. In order to perform finite element analyses, ANSYS software [10] is used. The intensity function and distribution of the slamming impact load are assumed the same as what considered in the Section 2.3. However, it should be emphasized that any other more detailed intensity function and distribution of slamming impact load can be easily implemented in the FEA.

The fitness evaluation process includes three steps. At first, coded chromosomes are decoded so that their relevant stacking sequences become clear. Then, geometrically non-linear finite element analysis is performed on the composite panel for the corresponding stacking sequence. For this purpose, both genetic algorithm code and finite element code are coupled in an especial procedure with each other. Finally, fitness of the chromosomes is to be evaluated. As explained earlier, the object of the present optimisation process is set to the minimisation of central deflection of the panel under lateral slamming impact load. Thus, the stacking sequence of plies that leads to a minimum deflection shows a maximum fitness. In order to determine the fitness of chromosomes, the following relationship is evaluated

$$
\text { Fitness value }{ }^{(c h)}=C-\text { Disp. }^{(c h)}
$$

The quantity Disp. in Eq. 2 represents the value of central deflection of the composite panel that is obtained from finite element analyses. The superscript (ch) in Eq. 2 also means that the corresponding values change for each chromosome. The parameter $C$ has a constant value throughout any time using of the optimisation process. In other words, the value of parameter $C$ is unchanged for all chromosomes in all generations. Based on above statements, for selection operator and also when evaluating the fitness function for chromosomes, the Eq. 1 is used. On the other hand, in order to show how the fitness function promotes along succeeding generations in the GA, the values of central deflection are represented as the fitness. The reason behind this is to show in a better way the process of minimisation of central deflection for the composite panel.

\section{APPLICATION EXAMPLES}

Different cases are investigated below in order to show how the optimum stacking sequence of the plies is determined for the slamming impact loaded composite panels applying the adopted GAFEA optimisation procedure. The cases include both symmetric and asymmetric stacking sequences for the composite panels having different numbers of plies.

\subsection{Optimum stacking sequences for symmetric four-ply composite panels}

In optimisation of symmetric four-ply composite panels under slamming loads, stacking sequence is assumed in the form of $[\alpha / \beta / \beta / \alpha]$. Optimisation subject is studied for three different dimensions of the panel; $1 \times 0.5 \mathrm{~m}, 1 \times 1 \mathrm{~m}$ and $1 \times 1.5 \mathrm{~m}$. An important matter that exists in solving such a problem is selection method of the parameters involved in the GA-FEA procedure. In order to avoid local optimum points, initial populations with 12 chromosomes are considered first, with 50

Latin American Journal of Solids and Structures 10(2013) $1043-1060$ 
generations. The parameters adopted are single-point crossover, mutation rate equal to 0.1 and crossover rate equal to 0.8. Fitness function evaluation in the GA for symmetric four-ply composite panels with different dimensions is shown in Fig. 7. As can be seen in the Fig. 7, with taking a relatively wide range of generations, it is assured that global optimum points inside the search spaces are reachable. However, this wide range of generations leads to a greater CPU time in the process of optimisation.

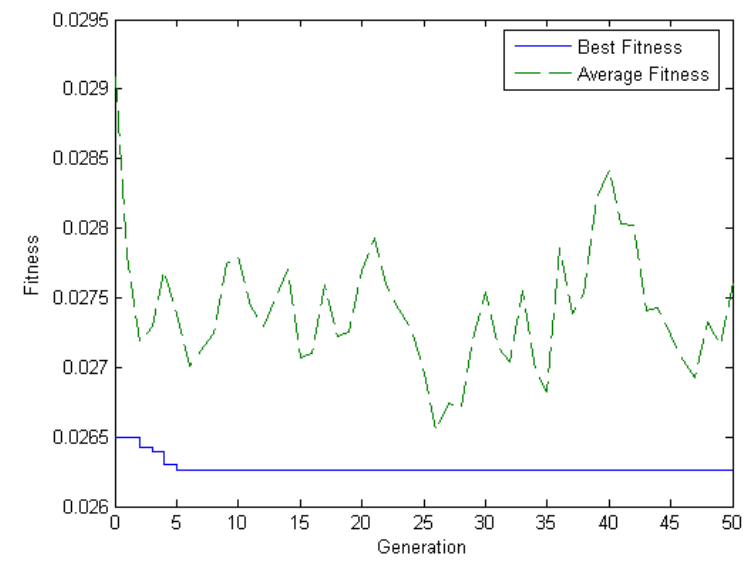

(a) Panel with the dimensions of $1 \times 0.5 \mathrm{~m}$

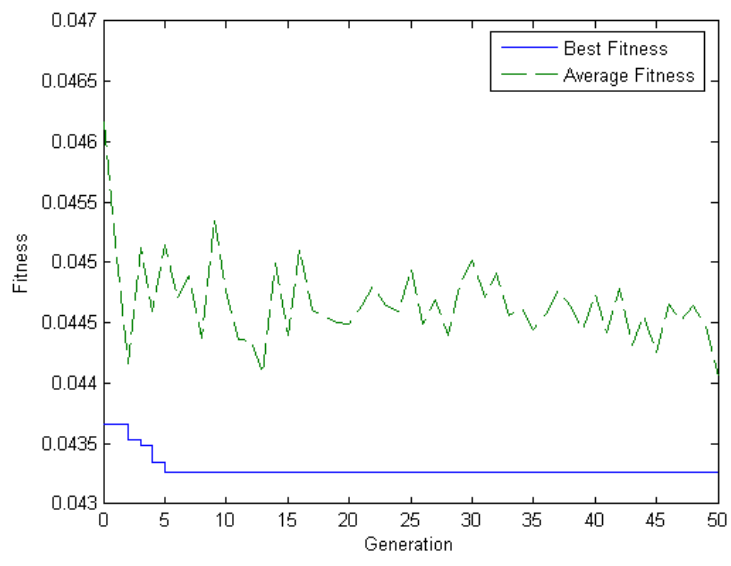

(b) Panel with the dimensions of $1 \times 1 \mathrm{~m}$

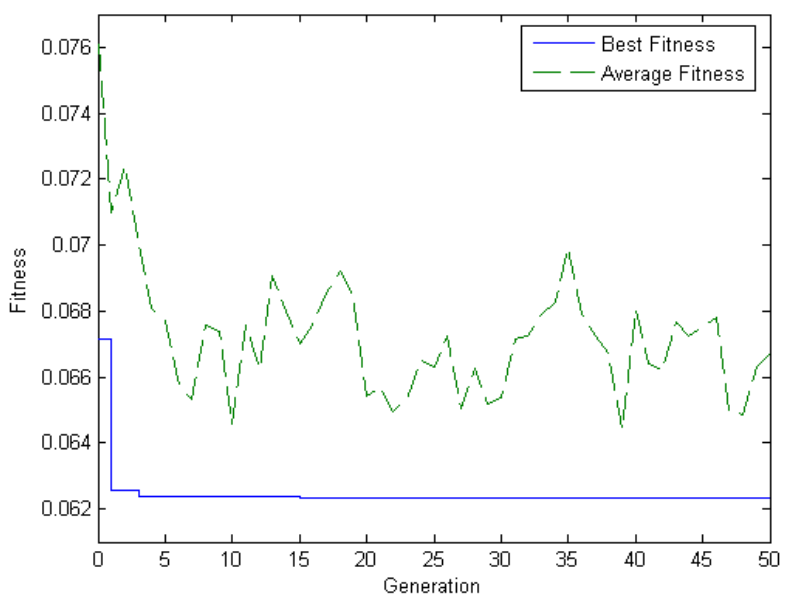

(c) Panel with the dimensions of $1 \times 1.5 \mathrm{~m}$

Figure 7 Fitness function evolution in the GA for symmetric four-ply composite panels.

Comparison of optimum stacking sequences for symmetric four-ply composite panels is given in Table 3. The results in Table 3 demonstrate that for any specific dimension of composite panel, two optimum stacking sequences are obtained for which the central deflection of the panel is exactly the same. On the other hand, it is interesting to see that the angles for the plies in the two optimum stacking sequences for any composite panel, have the same absolute values but symmetric to each other. Another remarkable matter is that for the composite panel of aspect ratio equal to 1, optimum stacking sequences are $[45 /-45 /-45 / 45]$ and $[-45 / 45 / 45 /-45]$. Since geometry, boundary 
condition and also loading condition of the panel are all symmetric in such a case, it is realised that balanced lay-ups have been obtained for the panel. This is another fact confirming the validity of adopted GA-FEA procedure.

Table 3 Comparison of optimum stacking sequences for symmetric four-ply composite panels

\begin{tabular}{ccc}
\hline Panel Dimension & Optimum Lay-up & Minimum Deflection $(\mathrm{m})$ \\
\hline \multirow{3}{*}{$1 \times 0.5 m$} & {$[-45 / 20 / 20 /-45]$} & 0.0252 \\
& {$[45 /-20 /-20 / 45]$} & \\
\hline & & \\
& {$[45 /-45 /-45 / 45]$} & 0.0437 \\
& {$[-45 / 45 / 45 /-45]$} & \\
\hline & \\
& {$[-45 / 60 / 60 /-45]$} & 0.0623 \\
\hline
\end{tabular}

\subsection{Optimum stacking sequences for asymmetric four-ply composite panels}

In this section, optimisation process is performed on the same panels investigated in previous section, but having asymmetric stacking sequences where the angles of plies are $[\alpha / \beta / \gamma / \lambda]$. For such a problem, it is certainly obvious that the search space is wide. Therefore, three-point crossover is implemented. During optimisation procedure, 40 generations of population are produced. The number of chromosomes in the initial population is increased from 12 to 20. Mutation and crossover rates are considered to be equal to 0.1 and 0.8, respectively. Evaluation of fitness function in the GA for asymmetric four-ply composite panels with different dimensions is shown in Fig. 8. Also, a comparison of optimum stacking sequences for asymmetric four-ply composite panels is provided in Table 4.

Table 4 Comparison of optimum stacking sequences for asymmetric four-ply composite panels

\begin{tabular}{ccc}
\hline Panel Dimension & Optimum Lay-up & Minimum Deflection $(\mathrm{m})$ \\
\hline $1 \times 0.5 m$ & $\begin{array}{l}{[-80 / 45 /-10 / 90]} \\
{[80 /-45 / 10 /-90]}\end{array}$ & 0.0255 \\
\hline $1 \times 1 m$ & $\begin{array}{l}{[-45 / 45 /-45 / 45]} \\
{[45 /-45 / 45 /-45]}\end{array}$ & 0.0433 \\
\hline $1 \times 1.5 m$ & {$[-45 / 45 / 60 /-60]$} & 0.0614 \\
\hline
\end{tabular}

Latin American Journal of Solids and Structures 10(2013) $1043-1060$ 


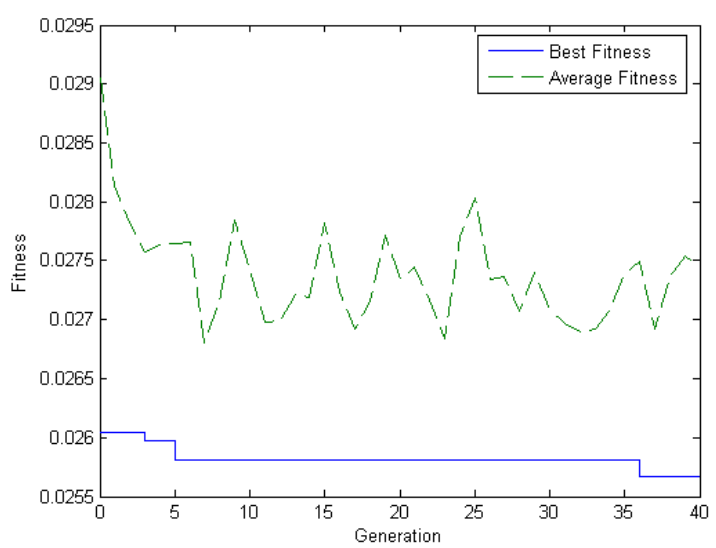

(a) Panel with the dimensions of $1 \times 0.5 \mathrm{~m}$

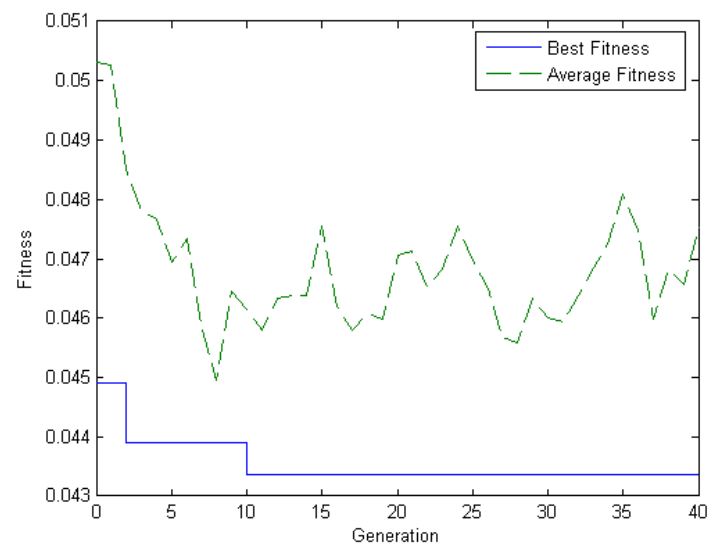

(b) Panel with the dimensions of $1 \times 1 \mathrm{~m}$

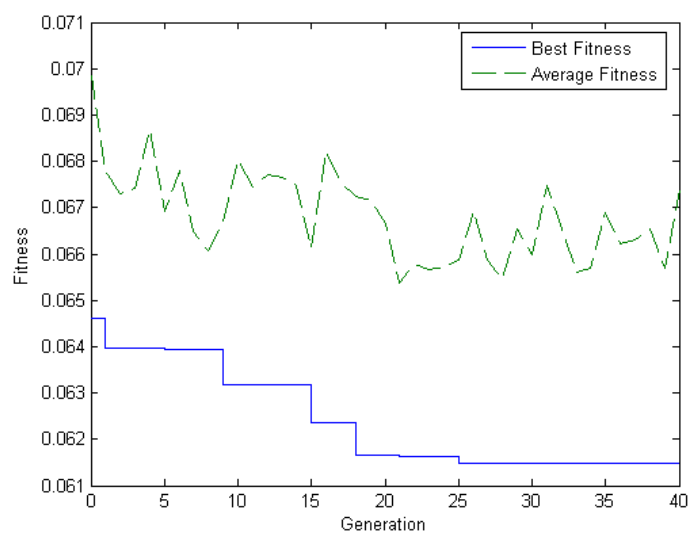

(c) Panel with the dimensions of $1 \times 1.5 \mathrm{~m}$

Figure 8 Fitness function evolution in the GA for asymmetric four-ply composite panels.

As can be seen from the results in Table 4, for any specific dimension of the composite panel in the case of asymmetric stacking sequence, two optimum lay-ups are obtained using the GA-FEA optimisation process. In both optimum lay-ups, the amount of central deflection is unchanged and furthermore, the angles of plies are same in absolute value but different in the sign. One interesting point that can be realised from comparison of the results reflected in Table 3 and Table 4 is that by changing symmetric stacking sequence to asymmetric one, the amount of central deflection is reduced by $1 \mathrm{~mm}$ for any specific dimension of the panel.

\subsection{Optimum stacking sequences for symmetric six-ply composite panels}

Optimisation is performed for the symmetric six-ply composite panels. Stacking sequence is of $[\alpha / \beta / \gamma / \gamma / \beta / \alpha]$ and also the panel has different dimensions of $1 \times 0.5 \mathrm{~m}, 1 \times 1 \mathrm{~m}$ and $1 \times 1.5 \mathrm{~m}$. For this purpose, 30 generations each having 16 chromosomes are utilised. Three-point crossover 
operator is applied in this process. Mutation and crossover rates are considered to be equal to 0.1 and 0.8 , respectively. Evaluation of fitness function in the GA for symmetric six-ply composite panels with different dimensions is shown in Fig. 9. Also, a comparison of optimum stacking sequences for symmetric six-ply composite panels is provided in Table 5 .

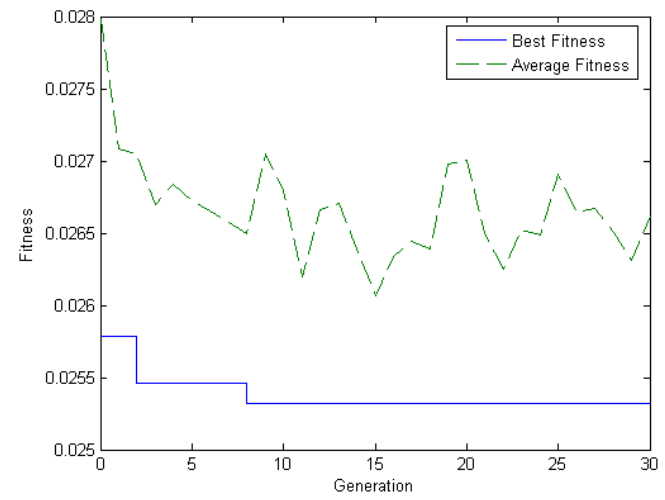

(a) Panel with the dimensions of $1 \times 0.5 \mathrm{~m}$

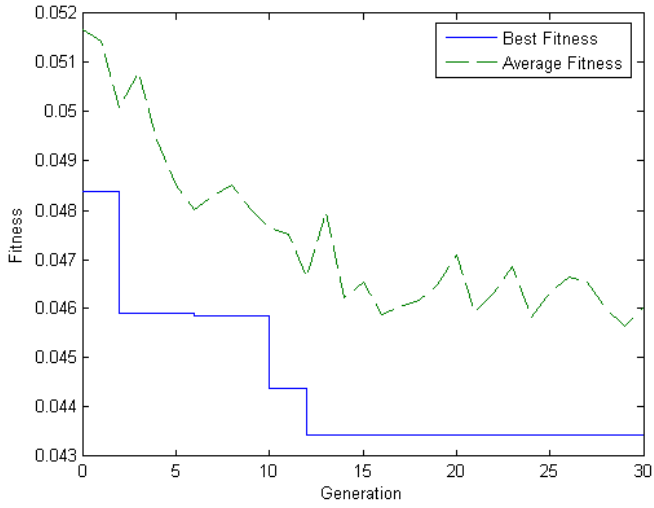

(b) Panel with the dimensions of $1 \times 1 \mathrm{~m}$

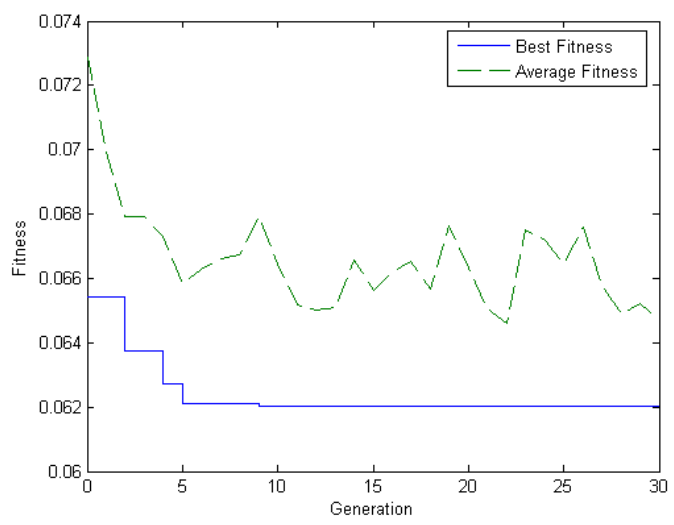

(c) Panel with the dimensions of $1 \times 1.5 \mathrm{~m}$

Figure 9 Fitness function evolution in the GA for symmetric six-ply composite panels.

Comparison of results for symmetric four-ply composite panels in Table 3 with those for symmetric six-ply composite panels in Table 5 shows that there are some similarities in the optimum stacking sequences for these two cases when the panel has the dimensions of $1 \times 1 \mathrm{~m}$ or $1 \times 1.5 \mathrm{~m}$. Besides, it is seen that by increasing the number of plies from 4 to 6 in case of composite panels with symmetric stacking sequence, the central deflection is reduced by amount of $0.3 \mathrm{~mm}$. 
Table 5 Comparison of optimum stacking sequences for symmetric six-ply composite panels

\begin{tabular}{ccc}
\hline Panel Dimension & Optimum Lay-up & Minimum Deflection $(\mathrm{m})$ \\
\hline $1 \times 0.5 m$ & {$[-80 / 45 /-10 /-10 / 45 /-80]$} & 0.0253 \\
& {$[80 /-45 / 10 / 10 /-45 / 80]$} & 0.0434 \\
\hline $1 \times 1 m$ & {$[45 /-45 /-45 /-45 /-45 / 45]$} \\
& {$[-45 / 45 / 45 / 45 / 45 /-45]$} & 0.062 \\
\hline
\end{tabular}

\subsection{Optimum stacking sequences for asymmetric six-ply composite panels}

Asymmetric six-ply composite panels are investigated in this section. The lay-up of the panels is assumed in the form of $[\alpha / \beta / \gamma / \lambda / \eta / \zeta]$. Again, since the search space in this case is very wide comparing with the other previous cases, three-point crossover operator is utilised. Initial population has 30 chromosomes and optimisation process is carried out for 50 generations. Mutation and crossover rates are considered to be equal to 0.15 and 0.9 , respectively. Fitness function promotion in the GA for asymmetric six-ply composite panels with different dimensions is shown in Fig. 10. Also, a comparison of optimum stacking sequences for asymmetric six-ply composite panels is provided in Table 6.

Table 6 Comparison of optimum stacking sequences for asymmetric six-ply composite panels

\begin{tabular}{ccc}
\hline Panel Dimension & Optimum Lay-up & Minimum Deflection $(\mathrm{m})$ \\
\hline \multirow{2}{*}{$1 \times 0.5 m$} & {$[-70 / 45 /-30 / 10 / 0 /-80]$} & 0.0249 \\
& {$[70 /-45 / 30 /-10 / 0 / 80]$} & \\
\hline \multirow{2}{*}{$1 \times 1 m$} & {$[-45 / 45 / 45 /-45 /-45 / 45]$} & 0.0432 \\
& {$[45 /-45 /-45 / 45 / 45 /-45]$} & \\
\hline \multirow{2}{*}{$1 \times 1.5 m$} & {$[-45 / 45 / 60 /-60 /-60 / 60]$} & 0.0608 \\
\hline
\end{tabular}




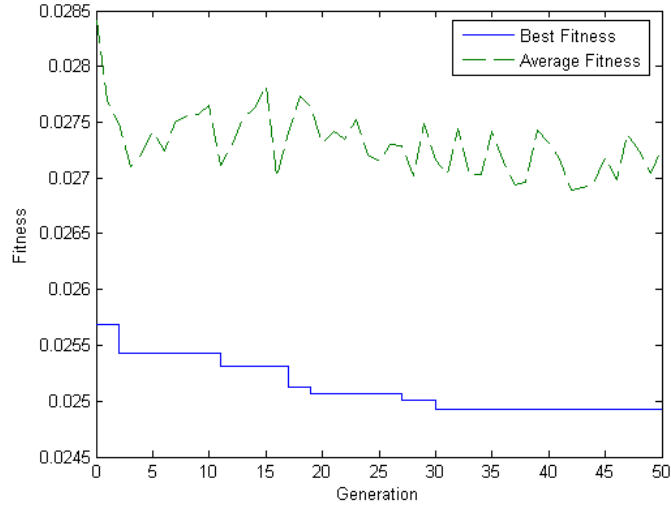

(a) Panel with the dimensions of $1 \times 0.5 \mathrm{~m}$

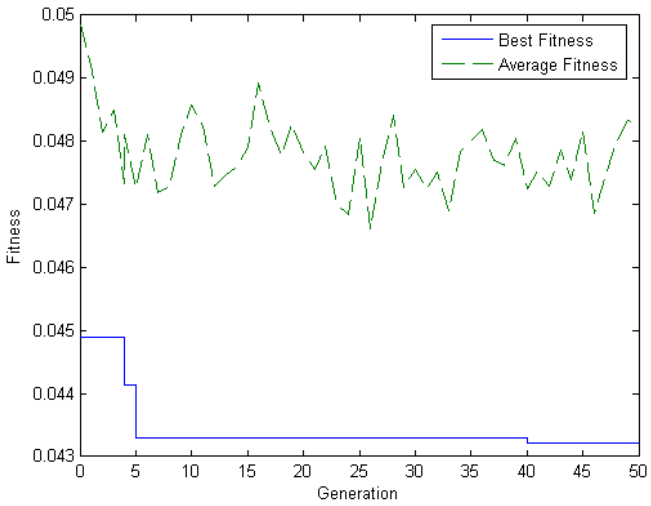

(b) Panel with the dimensions of $1 \times 1 \mathrm{~m}$

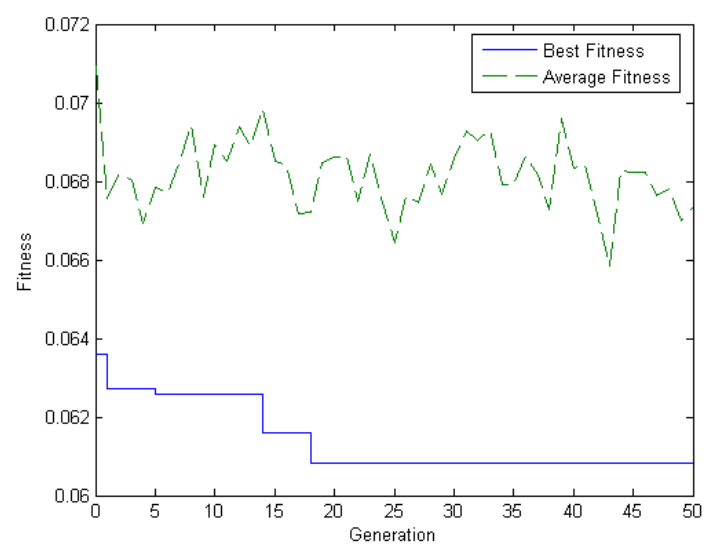

(c) Panel with the dimensions of $1 \times 1.5 \mathrm{~m}$

Figure 10 Fitness function evolution in the GA for asymmetric six-ply composite panels.

Comparing the results shown in Tables 4 and 6 reveals the fact that optimum stacking sequences for the composite panels with asymmetric lay-ups have some similarities. The optimum lay-ups for the asymmetric six-ply composite panels are of the form of $[\alpha /-\alpha /-\beta / \beta / \gamma /-\gamma]$. This conclusion should be considered with caution, since the slamming impact loads are not the only loads applying on the marine composite panels. Thus, the effects of all kinds of loads on the marine composite panels have to be taken into account when optimising their stacking sequences. Composite panels having asymmetric stacking sequences may exhibit bending and twisting deformations in addition to axial deformations under the action of in-plane axial loads. This is mainly due to the fact that their coupling matrix is not zero. Designers usually do not tend to use asymmetric stacking sequences in real practice, due to such properties. Comparing the results in Tables 5 and 6 shows that asymmetric stacking sequence for six-ply composite panels has caused a reduction of about $0.2 \mathrm{~mm}$ to $0.5 \mathrm{~mm}$ in the central deflection of the panels, with regard to the case of the same panels but having symmetric stacking sequence. Such an amount of reduction in the central deflection of the panel does not obviously satisfy the designers to adopt asymmetric stacking sequences in stead of symmetric ones. Another worth noting matter is that the panel of dimensions $1 \times 0.5 \mathrm{~m}$, does not 
obey the $[\alpha /-\alpha /-\beta / \beta / \gamma /-\gamma]$ lay-up. So such a proposal on the optimum lay-up in the case of asymmetric six-ply composite panels can not be generalised to different aspect ratios or sizes of the panels.

\section{CONCLUSIONS}

In this paper, an investigation was made into optimization of stacking sequence for the composite panels under slamming impact loads typically observed in the marine applications. The slamming impact load was considered to be evenly distributed over the panel surface, but varying in a triangular pulse manner with time. A genetic algorithm was coupled parallel to the finite element approach in order to solve the optimisation problem. Optimisation was performed for a variety of different cases changing in the panel dimensions as well as the lay-ups. The optimisation process was also validated in some way using the results for the case of a composite panel of dimensions $1 \times 1 \mathrm{~m}$ with symmetric stacking sequence. Out of the results obtained for various cases, it can be simply said that when the stacking sequence of the composite panel is asymmetric, then optimum lay-up follows the form of $[\alpha /-\alpha /-\beta / \beta / \gamma /-\gamma]$. Of course, there are some exceptions against this lay-up, especially when the aspect ratio of the panel is about 0.5 as in the case of the panel with dimensions of $1 \times 0.5 \mathrm{~m}$. For any specific number of plies, optimum asymmetric stacking sequence leads to a reduction in the central deflection of the panel in comparison with the case of applying optimum symmetric stacking sequence. The amount of reduction changes from case to case.

\section{References}

[1] Von Karman T. The impact on a seaplane floats during landing. Technical Notes for National Advisory Committee for Aeronautics, N.A.C.A. TN321, Washington, 1929.

[2] Wagner H. Uber stoss end an der oberfache von Flussigkeiten. Zeitschrift fur Angewandte Mathematik und Mechanik, 1932;12:193-215.

[3] Qin Z, Batra RC. Local slamming impact of sandwich composite hulls. International Journal of Solids and Structures 2009;46(10):2011-2035.

[4] Yong M, Falzon BG, Iannucci L. On the application of genetic algorithms for optimising composites against impact loading. International Journal of Impact Engineering, 2008;35(11):1293-1302.

[5] Holland J. Adaptation in natural and artificial systems. Ann Arbor, University of Michigan Press, 1975.

[6] Goldberg DE. Genetic algorithms in search, optimization and machine learning. Reading, Addison-Wesley, 1989.

[7] Mitchell M. An introduction to genetic algorithms. Cambridge, MIT Press, 1996.

[8] Callahan KJ, Weeks GE. Optimum design of composite laminates using genetic algorithms. Compos Eng, 1992;2(3):149-160.

[9] Rahul Chakraborty D, Dutta A. Optimization of FRP composites against impact induced failure using island model parallel genetic algorithm. Composites Science and Technology, 2005;65(13):2003-2013. 
[10] ANSYS 10.0 Reference Manual, ANSYS Inc.

[11] Kazanc Z, Mecitoglu Z. Nonlinear dynamic behavior of simply supported laminated composite plates subjected to blast load. J. of Sound and vibration, 2008;317(3-5):883-897.

[12] Gupta AD, Gregory FH, Bitting RL, Bhattacharya S. Dynamic analysis of an explosively loaded hinged rectangular plate. Computers \& Structures, 1987;26(1-2):339-344.

[13] Librescu L, Nosier A. Response of laminated composite flat panels to sonic boom and explosive blast loadings. AIAA Journal, 1990;28(2):345-352.

[14] Aslan Z, and Karakuzu R, Okutan B. The response of laminated composite plates under low-velocity impact loading. Composite Structures, 2003;59(1):119-127.

[15] Leite JPB, Topping BHV. Improved genetic operators for structural engineering optimization. In: Topping BHV, editor. Developments in neural networks and evolutionary computing for civil and structural engineering, Edinburgh, UK, 1995:143-69.

[16] Camp C, Pezesk S, Cao G. Optimized design of two-dimensional structures using a genetic algorithm. Journal of Structural Engineering, ASCE 1998;124(5):551-9.

[17] Chen T-Y, Chen C-J. Improvements of simple genetic algorithm in structural design. International Journal for Numerical Methods in Engineering 1997;40(7):1323-34.

[18] Hasancebi O, Erbatur F. Evaluation of crossover techniques in genetic algorithm based optimum structural design. In: Topping BHV, editor. Advances in engineering computational technology, Edinburgh, UK: CIVILCOMP Press, 1998:111-123.

[19] Suresh A, Mohammed A. An improved genetic algorithm for optimal design of large trusses. In: Topping BHV, editor. Advances in engineering computational technology, Edinburgh, UK: CIVILCOMP Press, 1998: 97-102.

[20] Muc A, Gurba W. Genetic algorithms and finite element analysis in optimization of composite structures. Composite Structures, 2001;54(2-3):275-281.

[21] Almeida FS, Awruch AM. Design optimization of composite laminated structures using genetic algorithms and finite element analysis. Composite Structures, 2009;88(3): 443-454. 